\title{
From sequences to enzymes: comparative genomics to study evolutionary conserved protein functions in marine microbes
}

Angelina Cordone ${ }^{1}$, Alessandro Coppola ${ }^{1}$, Angelica Severino ${ }^{1}$, Monica Correggia ${ }^{1}$, Matteo Selci $^{1}$, Antonio Cascone ${ }^{1}$, Costantino Vetriani ${ }^{2,3}$ and Donato Giovannelli ${ }^{1,2,4,5,6 *}$

1 Department of Biology, University of Naples Federico II, Naples, Italy

2 Department of Marine and Coastal Sciences, Rutgers University, New Brunswick, NJ, USA

3 Department of Biochemistry and Microbiology, Rutgers University, New Brunswick, NJ, USA

4 National Research Council - Institute of Marine Biological Resources and Biotechnologies CNR-IRBIM, Ancona, Italy

5 Earth-Life Science Institute, Tokyo Institute of Technology, Tokyo, Japan

6 Marine Chemistry \& Geochemistry Department - Woods Hole Oceanographic Institution, MA, USA

*corresponding author: donato.giovannelli@unina.it

Running Head: Comparative genomics of evolutionary conserved protein functions

\section{Abstract}

Comparative genomics is a research field that allows comparison between genomes of different life forms providing information on the organization of the compared genomes, both in terms of structure and encoded functions. Moreover, this approach provides a powerful tool to study and understand the evolutionary changes and adaptation among organisms. Comparative genomics can be used to compare phylogenetically close marine organisms showing different vital strategies and lifestyles and obtain information regarding specific adaptations and/or their evolutionary history. Here we report a basic comparative genomics protocol to extrapolate evolutionary information about a protein of interest conserved across diverse marine microbes. The outlined approach can be used in a number of different settings and might help to gain new insight into the evolution and adaptation of marine microorganisms.

Keywords: comparative genomics, ancestral proteins, evolution of functions, adaptations 


\section{Introduction}

Comparative genomics is a field of biological research in which the genome composition of different organisms is compared, both in terms of structure and encoded functions (1). Recent advances in sequencing technologies, modelling and molecular techniques have improved and accelerated the complete or near-complete genome sequencing of many organisms and microorganisms (2). These advances, together with the ability to recover near-complete Metagenome Assembled Genomes (MAGs) from the environment (3) have facilitated large scale comparative genomics and phylogenomics analysis. Comparative genomics aims to identify portions of DNA, RNA, or proteins conserved or divergent between different species, thus providing an important tool to study how evolution shaped the genomes of organisms (e.g., to identify ancestral and evolved functional traits) $(4,5)$.

In this contest, comparative genomics can be used to compare phylogenetically close organisms showing different vital strategies and lifestyles to analyze their adaptations through evolution $(6,7)$. For example, in marine microbiology, this approach has been used to compare gene functions between environmental and clinical relevant bacteria belonging to the Vibrio genus, thus identifying the unique and the common core functions of the pathogenic strains $(\boldsymbol{8}, \mathbf{9})$, or to identify conserved functions such as nitrate respiration or quorum sensing among deep-sea Epsilonproteobacteria (recently proposed to be reclassified as phylum Campylobacterota)(10) and their pathogenic relatives $(\mathbf{1 1}, 12)$.

Here, we show how to apply comparative genomics methods to study and analyze the evolution of proteins conserved across marine microbes adapted to different lifestyles (freeliving, epibionts, and obligate pathogens). Specifically, we showcase how the in silico identification of an unknown conserved protein and the study of its characteristics can be used to assess its evolutionary trajectory within defined groups of marine microorganisms. The results of this approach can be used downstream to inform heterologous expression experiments allowing comparative biochemical and functional characterization of the protein(s) (see Chapter [From sequences to enzymes: heterologous expression of genes from marine microbes]). The outlined approach can be used in a number of different settings and might help to gain new insight into the evolution and adaptation of marine microorganisms.

\section{Materials}

2.1 A computer with enough RAM to handle 3D visualization and phylogenetic tree construction might be required. A Linux based operating system (such as Ubuntu, Mint or Fedora) is preferred. Most programs used also work on Windows or Mac OS operating systems, but have not been tested with this protocol. An internet browser (Google Chrome, Firefox, etc), and a reliable internet connection are also necessary. Plots and statistical tests have been performed using the free software environment for statistical computing and graphics R (https://www.r-project.org/), and figure editing carried out using the free and 
open source vector graphics software Inkscape (https://inkscape.org/). Other software might be used in place of the ones described in this chapter and the ultimate selection rests upon the researcher.

2.2 Access to public genome repositories such as the National Genetic Sequence Database (GenBank, https://www.ncbi.nlm.nih.gov/search/) and the European Molecular Biology Laboratory European Bioinformatics Institute (EMBL-EBI, https://www.ebi.ac.uk/).

2.3 A local install of the NCBI Basic Local Alignment Search Tool (BLAST) or familiarity with the use of the online interface available at https://blast.ncbi.nlm.nih.gov/Blast.cgi.

2.4 A program for multiple sequence alignment. We used SeaView 4.0 (http://doua.prabi.fr/software/seaview) but several multi platform alternatives are available (13).

2.5 A phylogenetic reconstruction software to compute and manipulate phylogenetic trees. We used PhyLM 3.0, embedded in SeaView 4.0 (14) but several other well known options are available.

2.6 An open-source molecular visualization system for high quality three dimensional (3D) visualization and structure analysis of proteins. We used PyMOL for the analysis (15), but a number of other options are available.

2.7 A freely available program able to detect pockets and cavities of proteins of known 3D structure and to predict putative substrate-binding sites. In the present protocol we have used POcket-CAvity Search Application (POCASA) available online at http://g6altair.sci.hokudai.ac.jp/g6/service/pocasa/ (16).

2.7 A working knowledge of a comparative modeling server for 3D protein structures. For the present protocol we used the web based version of SWISS-MODEL available at https://swissmodel.expasy.org/ (17).

\section{Methods}

This protocol assumes that the protein of interest has been already identified through other means, for example large scale comparative genomic and pangenomic approaches (18), metagenomic analysis (3) or metabolic pathway reconstruction $(6,7,11)$. The identification of key proteins of interest is beyond the scope of this chapter.

\subsection{Retrieving closely related amino acid sequences from the database}

1. Using the Basic Local Alignment protein Search Tool (BLASTp) against the NCBI (or EMBL-EBI) database (19), manually search amino acid sequences closely related 
to the protein of interest from several representative strains belonging to the same taxonomic group or ecological group (see Note 1).

2. Download the amino acid FASTA files of all the examined proteins together with the relative accession numbers ( $\left.\mathrm{WP}_{-}\right)($see Note 2 and Note 3$)$.

\subsection{Retrieving 16S rRNA gene sequences for the selected organisms from the database}

1. Inspect the obtained amino acid sequences and identify the hosting organisms. The operation can be also done directly on the NCBI database by clicking on the organism name while on the GenBack view of the selected sequence.

2. Obtain and download the nucleotide FASTA files of the 16S rRNA genes from all the identified species, along with their respective accession numbers (NR_)(see Note 4).

\subsection{Amino acid sequence alignment and evaluation of conserved regions}

1. Align all the download amino acid sequences using the sequence alignment software and the algorithm of choice. We suggest the ClustalO alignment algorithm (20), available within SeaView (13). Several alternatives are available.

2. Manually inspect the obtained alignment and refine it if necessary.

3. Conserved amino acids sequence regions and motifs can be identified when aligning the sequence against a known template protein sequence.

4. The conserved regions can be annotated using BOXSHADE (version 3.21) available at https://embnet.vital-it.ch/software/BOX form.html.

\subsection{Nucleotide sequences alignment}

1. Align all the downloaded nucleotide sequences using the sequence alignment software and the algorithm of choice. We suggest the ClustalW alignment algorithm (20), available within SeaView (13). Several alternatives are available.

2. Manually inspect the obtained alignment and refine it if necessary.

\subsection{Phylogenetic trees construction}

1. Identify the best fitting amino acid or nucleotide substitution to use for tree generation from the alignment. We suggest ProtTest3 (21) for the amino acid sequences and JModelTest (22) for the nucleotide sequences. 
2. Once the best substitution model has been identified, construct the maximumlikelihood phylogenetic tree for both the protein sequence and the 16S rRNA gene sequences using the identified parameters. We use PhyML for this purpose (14), selecting alternative likelihood ratio tests (a-LRT) for evaluating branch support.

3. Root the obtained tree according to a pre-selected strategy. Appropriate rooting is required to infer robust evolutionary information (see Note 5). The obtained trees can be efficiently visualized and manipulated using tools like FigTree (23) and the Interactive Tree Of Life tool (ITOL)(24).

\subsection{Phylogenetic trees reconciliation (optional step)}

The phylogenetic trees reconstructed as described in the previous section can be directly reconciled to obtain more reliable information regarding potential horizontal gene transfer events and gene shuffling across taxa. Tree reconciliation can be done in a number of different ways. We take advantage of the "Reconcile tree" function of PhyML (see Note 6).

1. Open the functional protein and the 16S rRNA gene tree with PhyML in the SeaView program.

2. Organize the $16 \mathrm{~S}$ rRNA gene tree as preferred, being sure to root the tree accordingly.

3. Once the 16S rRNA gene tree has been organized according to selected criteria, select the "reconcile tree" function from the PhyML menu, and reconcile the protein tree to the $16 \mathrm{~S}$ rRNA tree.

4. Save both trees in a vector format. We routinely use .svg or .eps to save the visualization of the trees.

5. The obtained vector files can be imported into a vector graphic software such as Inkscape. The tree can now be arranged side by side.

6. To highlight changes in the branching of the organismal and protein trees, segments connecting genes originating from the same organisms can be drawn. Also, coloring according to a specific factor, such as phylogeny, ecological adaptations or specific physiological traits can be used (Figure 2).

\subsection{Identification of the genomic context for the protein-coding gene of interest}

Proteins are often part of complex molecular machineries created by multiple subunits that can also be associated with regulatory proteins and maturation chaperones. The analysis of the gene context of a protein can provide additional information regarding its putative function, its regulations and its evolutionary history. Several alternative approaches can be used to investigate the genomic context of a protein-coding gene across multiple organisms. Here we highlight one such possible approach. 
1. The genomic context of the protein-coding gene of interest of each organism under investigation can be directly inspected using the Genome portal of the NCBI database.

2. The operonic structure can be investigated for each genome using either FGenesB (http://www.softberry.com/berry.phtml?

topic $=$ fgenesb\&group $=$ programs\&subgroup $=$ gfindb), searching for the organisms of interest in the pre-computed ProOpDB (25), through Patric (26), BioCyc.org (27) or the CoGe GenomeEvolution web service (28) (Figure 3) (see Note 7).

3. The obtained genome context information can be used to directly compare operonic structures and genes upstream and downstream from the gene of interest, providing information on the evolutionary history of the entire molecular machinery involved in the process under investigation.

\subsection{Protein 3D structure homology modeling}

Homology modeling of the protein of interest is carried out through a computational analysis on Swiss-Model (17). Prior to starting homology modelling, verify how many 3D structures have been resolved for closely related proteins. The modelling results will be more accurate if the number of protein structures available is higher. It is possible to access all known protein structures through the RCSB Protein Data Bank database (https://www.rcsb.org/).

1. Upload all the amino acid sequences for the selected proteins to the Swiss-Model interface (https://swissmodel.expasy.org/interactive), which performs an homology search of the structure database (see Note 8).

2. Once the best match for each protein to be modelled has been identified, proceed with the modelling and download all the protein 3D models in PDB format.

3. Calculate the root-mean-square deviation values (RMSD) using the PyMol program (15) between the protein of interest and the selected proteins representing the cluster being investigated. The smaller the RMSD the closer the two structures are in 3dimensional space.

4. Use the ColorByRMSD plugin in PyMol to obtain visual information regarding the distribution of the RMSD between two protein structures according to the structure atoms deviation (Figure 4) (see Note 9).

5. Additional measurements can be done on the obtained models, and compared across the organisms under investigation. For example, in the case of enzymes with an active pocket, the protein ligand binding sites and the volume of the pocket can be predicted using the POCASA program (16). Molecular docking experiments can be carried out using the ligand of interest and the UCSF Chimera program (29) with the extension AutoDock-Vina (30) (see Note 10). 


\section{Notes}

1. The boundaries of the group of interest can be defined either based on taxonomic, phenotypic or ecological characteristics. The way the different organisms are grouped can and need to be changed according to the research question. For example, taxonomically related groups of organisms can be used to investigate relationships between vertical inheritance and adaptations, while groups based on similar lifestyle and phenotype can be used to study the protein adaptations to specific ecological niches.

2. We suggest that you rename the obtained sequences with a meaningful name that is human readable. We have found it useful to use the first letter of the genus name followed by the first three letters of the species name, for example Ddes for Desulfovibrio desulfuricans, to uniquely identify the organism from which each sequence originated. Other solutions are possible and might be required when comparing strains of the same species or in similar cases.

3. Keeping a table with all the organisms information, accession numbers and bibliographic references will help in the downstream processing of the results. The same table can also be annotated with taxonomic, physiologic, metabolic, and ecological information to help interpret the results.

4. While it is possible to obtain information about the evolutionary history of the protein of interest with respect to the organisms comparing the functional protein and the organismal tree, to be able to perform a couple reconstruction it is necessary that for each functional protein the corresponding $16 \mathrm{~S}$ rRNA gene is also downloaded.

5. Rooting phylogenetic trees might be of primary importance depending on the type of hypothesis being tested in the study. Trees can be rooted using a number of different approaches (31). We have found that robust inferences might be obtained when selecting a rooting strategy based on established outgroups for the organismal (16S rRNA) tree, and using the corresponding proteins from those organisms in the protein tree. This strategy might however lead to misleading results if the functional protein has undergone lateral gene transfer in the outgroup only, or if it is not conserved in the outgroup.

6. Tree reconciliation in PhyML is based on the sequence name. To be carried out it is necessary that both the protein and the 16S rRNA sequences have the exact same name.

7. While all the outlined software can provide information regarding the gene context, manual inspection is often required together with multiple BLAST searches to confirm the findings. Additionally, the best quality figures are obtained using a vector based graphic software such as Inkscape and drawing the genes to scale.

8. The Swiss-Model interface will return multiple targets ranked from best to worst. Before selecting the best target for modelling, be sure to inspect the model parameters for the goodness of fit, such as the coverage and the identity. The higher these parameters the better the computed model.

9. RMSD if computed pairwise for all the available proteins can be used to generate a RMSD matrix to be used as input distance matrix in the $\mathrm{R}$ statistical software to 
obtain a distance based tree from the protein structures. This can be then reconciled to the phylogenetic tree obtained from the amino acid sequences. The patristic distances obtained from the two trees can be plotted as a scatter plot to visualize discontinuities in the relationship between sequence evolution and structural changes.

10. The obtained information like pocket volume, docking score, etc... can all be used to relate information regarding the protein $3 \mathrm{D}$ structure to the phylogenetic analysis providing insight into the evolutionary history of the protein.

\section{Acknowledgement}

This work was partially supported by the European Research Council (ERC) under the European Union's Horizon 2020 research and innovation programme (grant agreement No. 948972) and by NSF grants MCB 15-17567 and IOS 19-51690. 


\section{References}

1. Tripathi A (2019) Comparative Genomics A New Arena of Biological Research: A Review. 7

2. Mardis ER (2017) DNA sequencing technologies: 2006-2016. Nat Protoc 12:213-218

3. Malmstrom RR and Eloe-Fadrosh EA Advancing Genome-Resolved Metagenomics beyond the Shotgun. mSystems 4:e00118-19

4. Ellegren H (2008) Comparative genomics and the study of evolution by natural selection. Mol Ecol 17:4586-4596

5. Hardison RC (2003) Comparative Genomics. PLOS Biol 1:e58

6. Giovannelli D and Vetriani C (2017) From extreme environments to human pathogens: an evolutionary journey. The Biochemist 39:4-9

7. Giovannelli D, Sievert SM, Hügler M, et al (2017) Insight into the evolution of microbial metabolism from the deep-branching bacterium, Thermovibrio ammonificans. eLife 6:e18990

8. Hasan NA, Grim CJ, Haley BJ, et al (2010) Comparative genomics of clinical and environmental Vibrio mimicus. Proc Natl Acad Sci U S A 107:21134-21139

9. Le Roux F, Wegner KM, Baker-Austin C, et al (2015) The emergence of Vibrio pathogens in Europe: ecology, evolution, and pathogenesis (Paris, 11-12th March 2015). Front Microbiol 6:830

10. Waite DW, Chuvochina MS, and Hugenholtz P (2019) Road Map of the Phylum Campylobacterota, In: Bergey's Manual of Systematics of Archaea and Bacteria, pp. 111 American Cancer Society

11. Vetriani C, Voordeckers JW, Crespo-Medina M, et al (2014) Deep-sea hydrothermal vent Epsilonproteobacteria encode a conserved and widespread nitrate reduction pathway (Nap). ISME J 8:1510-1521

12. Pérez-Rodríguez I, Bolognini M, Ricci J, et al (2015) From deep-sea volcanoes to human pathogens: a conserved quorum-sensing signal in Epsilonproteobacteria. ISME J 9:1222-1234

13. Gouy M, Guindon S, and Gascuel O (2010) SeaView Version 4: A Multiplatform Graphical User Interface for Sequence Alignment and Phylogenetic Tree Building. Mol Biol Evol 27:221-224

14. Guindon S, Dufayard J-F, Lefort V, et al (2010) New Algorithms and Methods to 
Estimate Maximum-Likelihood Phylogenies: Assessing the Performance of PhyML 3.0. Syst Biol 59:307-321

15. Schrodinger LLC (2010) The PyMOL molecular graphics system, version 1.5. 0.4. Schrodinger LLC

16. Yu J, Zhou Y, Tanaka I, et al (2010) Roll: a new algorithm for the detection of protein pockets and cavities with a rolling probe sphere. Bioinforma Oxf Engl 26:46-52

17. Waterhouse A, Bertoni M, Bienert S, et al (2018) SWISS-MODEL: homology modelling of protein structures and complexes. Nucleic Acids Res 46:W296-W303

18. Delmont TO and Eren AM (2018) Linking pangenomes and metagenomes: the Prochlorococcus metapangenome. PeerJ 6:e4320

19. Altschul SF, Madden TL, Schäffer AA, et al (1997) Gapped BLAST and PSI-BLAST: a new generation of protein database search programs. Nucleic Acids Res 25:3389-3402

20. Sievers F, Wilm A, Dineen D, et al (2011) Fast, scalable generation of high-quality protein multiple sequence alignments using Clustal Omega. Mol Syst Biol 7:539

21. Darriba D, Taboada GL, Doallo R, et al (2011) ProtTest 3: fast selection of best-fit models of protein evolution. Bioinformatics 27:1164-1165

22. Posada D and Crandall KA (1998) Modeltest: testing the model of DNA substitution. Bioinformatics 14:817-818

23. Rambaut A (2009) FigTree v1. 3.1. Httptree Bio Ed Ac Uksoftwarefigtree

24. Tung M-C, Hsu B-M, Tao C-W, et al (2013) Identification and significance of Naegleria fowleri isolated from the hot spring which related to the first primary amebic meningoencephalitis (PAM) patient in Taiwan. Int J Parasitol 43:691-696

25. Taboada B, Ciria R, Martinez-Guerrero CE, et al (2012) ProOpDB: Prokaryotic Operon DataBase. Nucleic Acids Res 40:D627-D631

26. Wattam AR, Abraham D, Dalay O, et al (2014) PATRIC, the bacterial bioinformatics database and analysis resource. Nucleic Acids Res 42:D581-D591

27. Karp PD, Billington R, Caspi R, et al (2019) The BioCyc collection of microbial genomes and metabolic pathways. Brief Bioinform 20:1085-1093

28. Lyons E, Pedersen B, Kane J, et al (2008) Finding and Comparing Syntenic Regions among Arabidopsis and the Outgroups Papaya, Poplar, and Grape: CoGe with Rosids. Plant Physiol 148:1772-1781

29. Pettersen EF, Goddard TD, Huang CC, et al (2004) UCSF Chimera-A visualization system for exploratory research and analysis. J Comput Chem 25:1605-1612

30. Trott O and Olson AJ (2010) AutoDock Vina: improving the speed and accuracy of 
docking with a new scoring function, efficient optimization and multithreading. J Comput Chem 31:455-461

31. Huelsenbeck JP, Bollback JP, and Levine AM (2002) Inferring the Root of a Phylogenetic Tree. Syst Biol 51:32-43

32. Kavermann H, Burns BP, Angermüller K, et al (2003) Identification and Characterization of Helicobacter pylori Genes Essential for Gastric Colonization. J Exp Med 197:813-822

33. Giovannelli D, Chung M, Staley J, et al (2016) Sulfurovum riftiae sp. nov., a mesophilic, thiosulfate-oxidizing, nitrate-reducing chemolithotrophic Epsilonproteobacterium isolated from the tube of Riftia pachyptila, a deep-sea hydrothermal vent tube worm. Int J Syst Evol Microbiol Submitted 


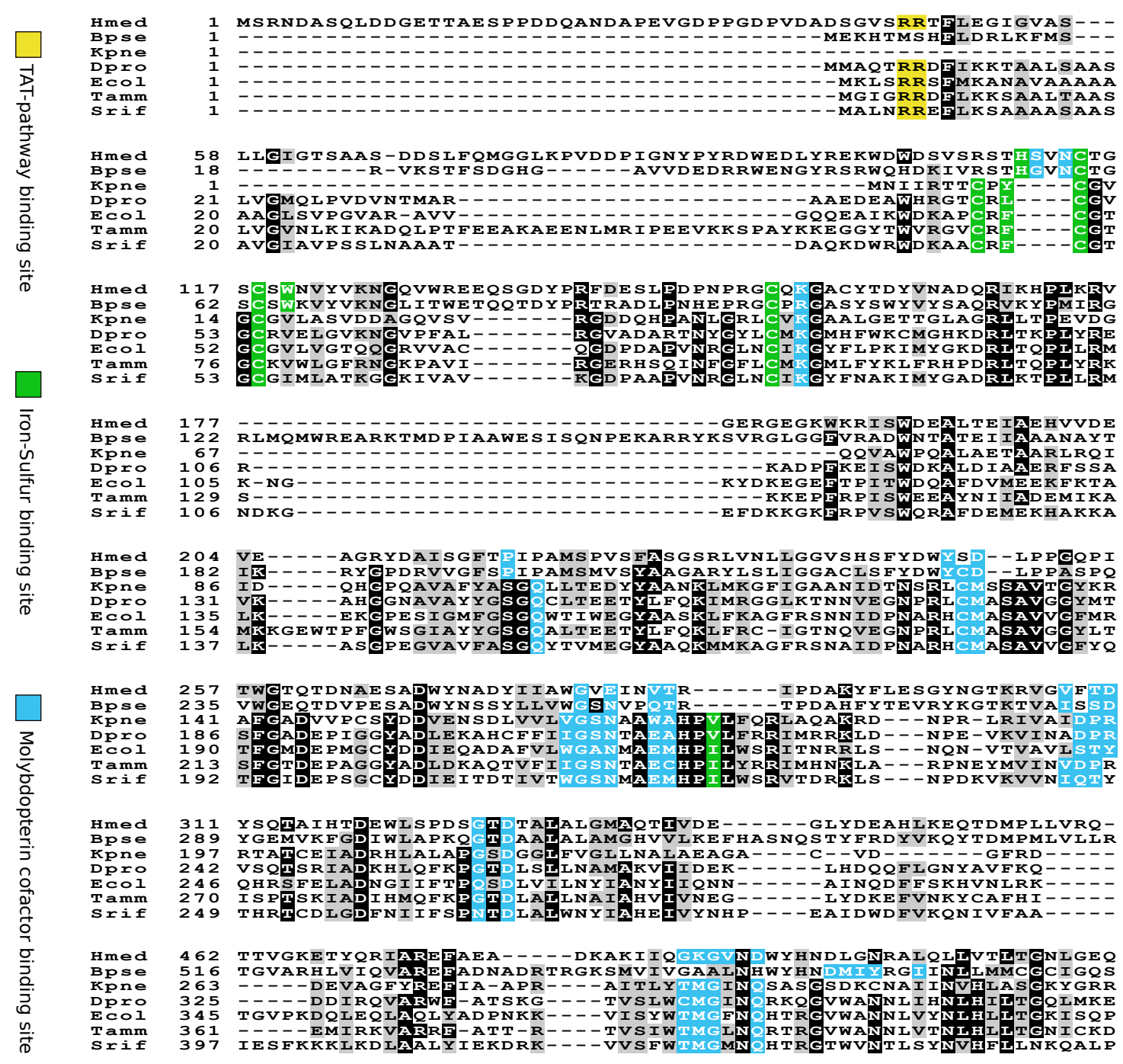

Figure 1. A multiple sequence alignment of amino acid sequences encoding for the catalytic subunit of different nitrate reductases. Hmed - Haloferax mediterranei pNarG; Bpse Burkholderia pseudomallei NarG; Kpne - Klebsiella pneumoniae NasA; Dpro Desulfobulbus propionicus monomeric NapA (NapC-independent); Ecol - Escherichia coli dimeric NapA (NapC-dependent); Tamm - Thermovibrio ammonificans monomeric NapA (NapC-dependent); Srif - Sulfurovum riftiae dimeric NapA (NapC-independent). The conserved regions are highlighted on the basis of the information available in the literature. 


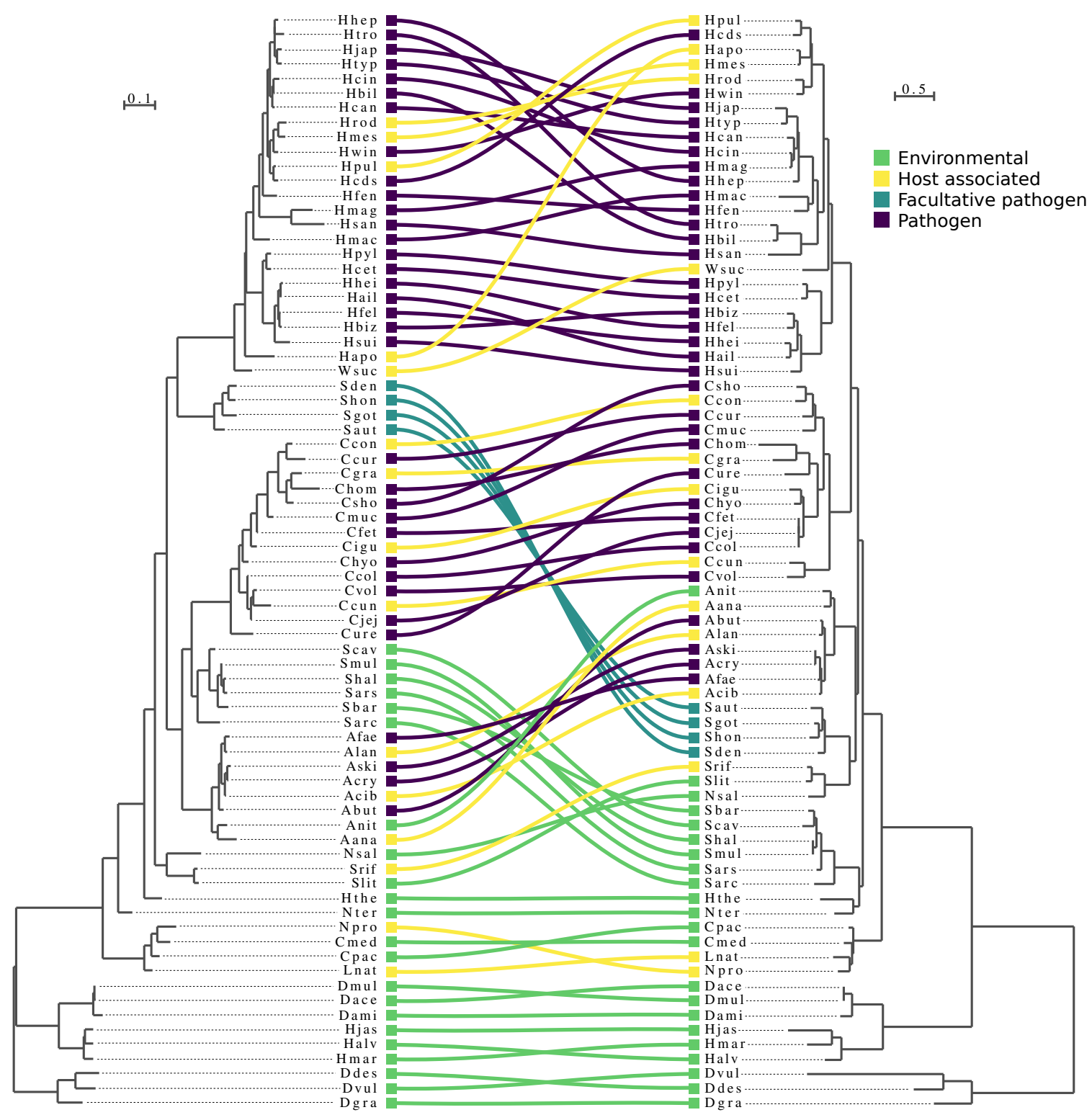

Figure 2. Example of reconciled tree between the 16S rRNA gene and the PrtC-like gene from 75 representative species within the class Epsilonproteobacteria (recently proposed to be reclassified as the phylum Campylobacterota) (10). The PrtC-like protein is a putative protease conserved in all Epsilonproteobacteria, including those found at deep-sea hydrothermal vents, and important for the virulence of the human pathogen Helicobacter pylori (32). 


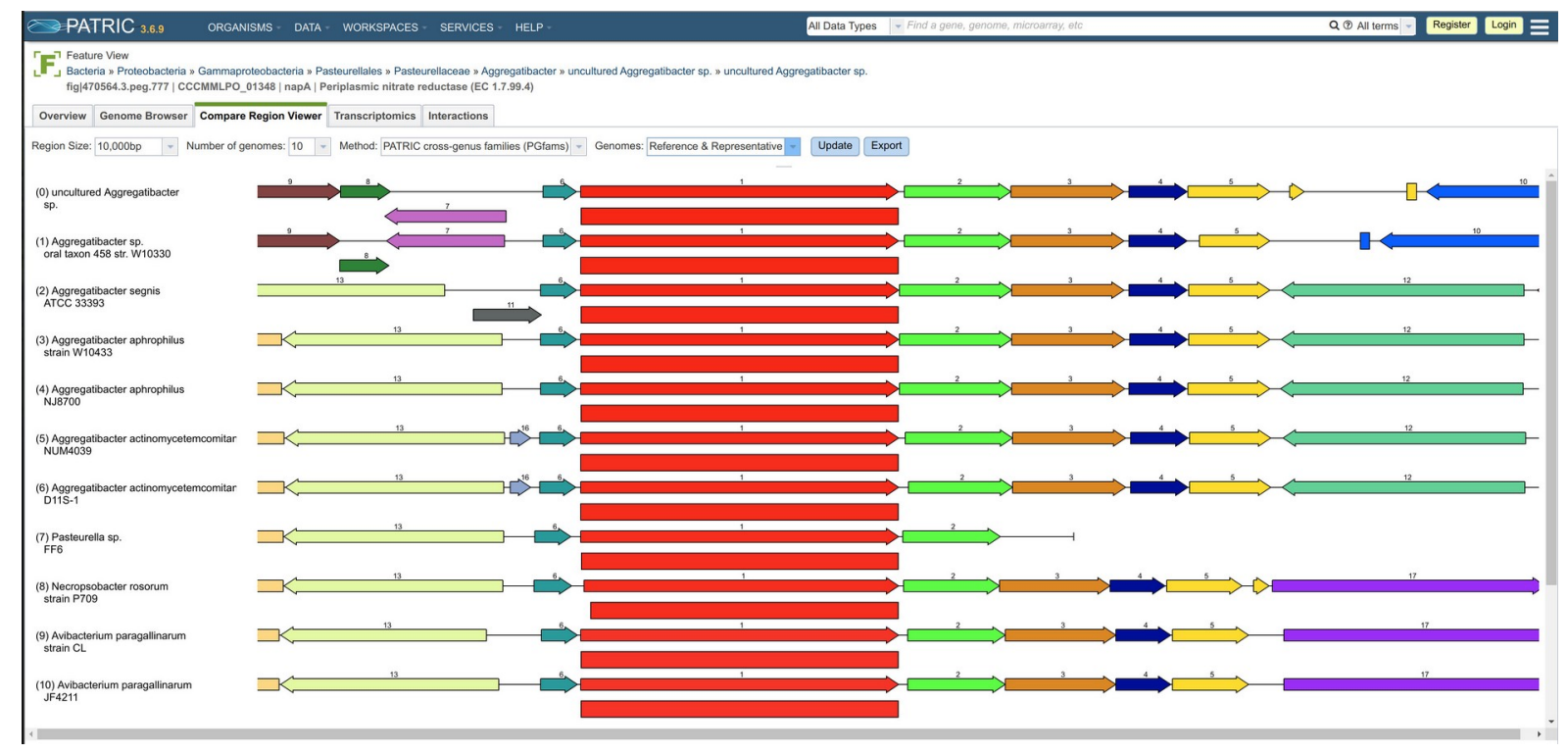

Figure 3. Example of screenshot obtained from Patric and relative to the gene context of the periplasmic nitrate reductase (napA) gene. 


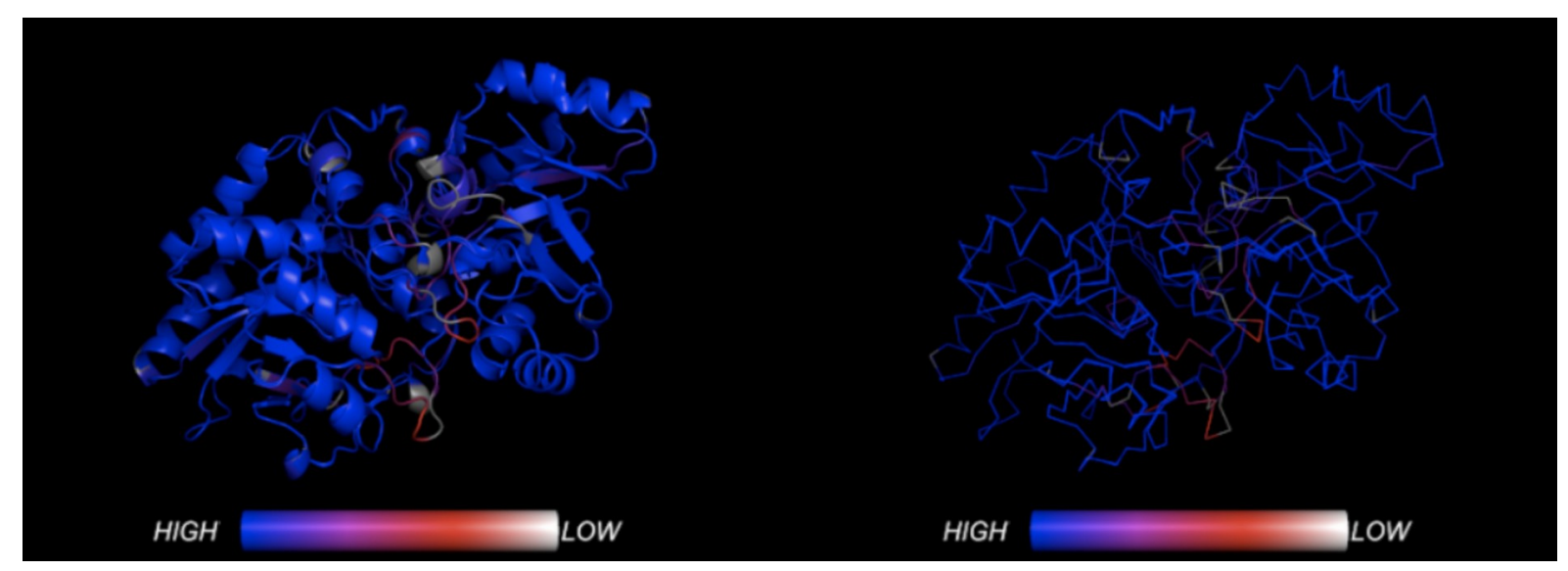

Figure 4. Superimposition of the PrtC-like protein of Sulfurovum riftiae (an epibionts of the deep-sea hydrothermal vent tubeworm Riftia pachyptila) (33) and Helicobacter pylori (an obligate human pathogen) colored by root-mean-square deviation values. 